PROCEEDINGS OF THE

AMERICAN MATHEMATICAL SOCIETY

Volume 130, Number 1, Pages 205-212

S 0002-9939(01)06004-X

Article electronically published on May 22, 2001

\title{
A NOTE ON GROUPS DEFINABLE IN DIFFERENCE FIELDS
}

\author{
PIOTR KOWALSKI AND A. PILLAY \\ (Communicated by Carl G. Jockusch, Jr.)
}

\begin{abstract}
We prove that a group definable in a model of $A C F A$ is virtually definably embeddable in an algebraic group. We give an improved proof of the same result for groups definable in differentially closed fields. We also extend to the difference field context results on the unipotence of definable groups on affine spaces.
\end{abstract}

\section{INTRODUCTION AND PRELIMINARIES}

In this paper we record some observations around groups definable in difference fields. We were motivated by a question of Zoe Chatzidakis as to whether any group definable in a model of $A C F A$ is virtually definably embeddable in an algebraic group. We give a positive answer. Among the possibly "new" ingredients is the (stable) group configuration theorem in the $*$-definable category. The embeddability result for groups definable in $A C F A$ of finite $S U$-rank was already noted in [2], more or less by saying that the proof in [5] for groups definable in pseudofinite fields goes through.

We also take the opportunity to give an improved treatment of the analogous theorem for differentially closed fields (avoiding the category of $*$-definable groups).

Finally we adapt results from [6] and [1] about the unipotence of differential groups on affine spaces to the difference field context.

The results here have little to do with either automorphisms or derivations and could be presented in a suitable axiomatic framework.

As usual we work in a saturated model $\bar{M}$ of a complete theory $T$. We work freely in $\bar{M}^{e q}$. We assume knowledge of stability theory, stable groups and the more general versions for simple theories. See 8], [10]. We will also use a result from [1]. We also require a few easy observations around stabilisers and generics which are not explicit in the literature, so we give them now.

Definition 1.1. Assume $T$ is simple, and $G$ is a type-definable group over a model $M$.

(i) Let $H$ be a type-definable over $M$ subgroup of $G$ and $X$ a right coset of $H$ in $G$, also defined over $M$. Let $q(x)$ be a complete type over $M$ of an element of $X$. We call $q(x)$ a generic type of $X$ (over $M$ ) if for some realisation $b$ of $q$ and some element $c$ of $X$ independent from $b$ over $M, t p\left(b . c^{-1} / M \cup\{c\}\right)$ is a generic type of

Received by the editors April 5, 2000 and, in revised form, May 16, 2000.

2000 Mathematics Subject Classification. Primary 03C60.

The first author was supported by grant KBN 2 PO3A 02018.

The second author was supported by an NSF grant. 
$H$. (Intuitively $q(x)$ is a translate of a generic type of $H$.) Analogously if $X$ is a left coset of $H$ in $G$.

(ii) Assume $q(x), r(x)$ are complete types over $M$ of elements of $G$. By $S(q, r)$ we mean the (type-definable over $M$ ) set consisting of those $a \in G$ such that for some realization $b$ of $q$, independent with $a$ over $M$, a.b realises $r$ and is independent with $a$ over $M$.

Lemma 1.2 ( $T$ simple and $G$ type-definable over $M)$. (i) With the notation of (i) above, $q(x)$ is a generic type of $X$ if and only if for any realization $b$ of $q$ and for any $a \in H$ independent of $b$ over $M$, a.b is independent from a over $M$.

(ii) With the notation of (i) above, let $X$ be the right coset generated by $S(q, r)$. Then $X$ is type-definable over $M, X$ is a right coset of (left) $\operatorname{Stab}(r)$ and $t p(a / M)$ is a generic type of $X$. Similarly, the left coset $Y$ generated by $S(q, r)$ is type-definable over $M$, is a left coset of (left) $\operatorname{Stab}(q)$ and tp $(a / M)$ is a generic type of $Y$.

(iii) Suppose $a, b, c$ are elements of $G$ pairwise independent over $M$ such that $a . b=c$ and $\operatorname{tp}(b / M)=t p(c / M)=q$. Let $H=($ left $)$ Stab $(q)$. Then H.b is typedefinable over $M$ and $t p(b / M)$ is a generic type of H.b.

Proof. (i) is routine.

(ii) Choose $d \in \operatorname{Stab}(r)$, with $d$ independent of $a$ over $M$. Without loss of generality, $d$ is independent of $\{a, b, c\}$ over $M$ and moreover by the Independence Theorem we may assume that $d . c=c^{\prime}$ realises $r$ and is independent from $d$ over $M$. So $d . a=c . b^{-1}$ is independent from $d$ over $M$. By (i), $t p(a / M)$ is a generic of $X$.

(iii) Note that by (ii) $t p(a / M)$ is a generic type of $H$. Let $X=H . b$. Clearly $X=H . c$ also. We first show that $X$ is $M$-invariant, hence type-definable over $M$ : Let $c^{\prime}$ realise $q$ independently from $c$ over $M$. By the Independence Theorem there is $b^{\prime}$ such that $t p\left(c, b^{\prime} / M\right)=t p\left(c^{\prime}, b^{\prime} / M\right)=t p(c, b / M)$. Hence $b^{\prime} \in H . c \cap H . c^{\prime}$, whereby $H . c=H . c^{\prime}$ (as $H$ is $M$ invariant). This is enough. Now by definition, $q$ is a generic type of $X$.

\section{Groups in Stable theories And Simple theories}

By a $*$-tuple we simply mean a possibly infinite tuple $\left(a_{i}\right)_{i \in I}$ of elements of $\bar{M}^{e q}$ (where the index set $I$ has cardinality less than that of $\bar{M}$ ). By a $*$-definable (over $A$ ) set we mean a collection of $*$-tuples (each tuple being indexed by some fixed $I$ ), which is the set of realizations of a partial type $\Sigma\left(x_{i}\right)_{i \in I}$ over a set $A$ of parameters. By a $*$-definable (over $A$ ) group we mean a group $G$ such that both $G$ and the graph of multiplication are $*$-definable (over $A$ ) sets. Similarly we have the notion of a $*$-definable homogeneous space $(G, S)$. If the underlying set of $G$ consists of finite tuples, we will say that $G$ is finitary. A finitary $*$-definable group is what is usually called a type-definable group. If $T$ is stable the theory of generic types, etc., passes over to $*$-definable groups. Moreover Hrushovski proves in [4] that (for $T$ stable) any $*$-definable group (homogeneous space) is $*$-definably isomorphic to a projective limit of finitary $*$-definable groups (homogeneous spaces). In the totally transcendental case, this becomes a projective limit of definable groups (homogeneous spaces). Our first general result is the group configuration theorem (as stated in Theorem 4.5 of [8]) generalized to $*$-definability. This must be wellknown to anybody who has thought about it. There is only one delicate point that has to be take care of which we will point out below. As a matter of notation when 
we say, for example, that a $*$-tuple $c=\left(c_{i}\right)_{i \in I}$ is contained in $\operatorname{acl}(A)$, we mean that each $c_{i} \in \operatorname{acl}(A)$.

Proposition 2.1. Let $M$ be a saturated model of the stable theory $T$. Let $a, b, c, x$, $y, z$ be *-tuples of length strictly less than the cardinality of $M$. Suppose that the following are true:

(i) $\operatorname{acl}(M, a, b)=\operatorname{acl}(M, a, c)=\operatorname{acl}(M, b, c)$.

(ii) $\operatorname{acl}(M, a, x)=\operatorname{acl}(M, a, y)$ and $C b(\operatorname{stp}(x, y / M, a))$ is interalgebraic with a over $M$.

(iii) As in (ii) with $b, z, y$ in place of $a, x, y$.

(iv) As in (ii) with $c, z, x$ in place of $a, x, y$.

(v) Other than $\{a, b, c\},\{a, x, y\},\{b, z, y\}$ and $\{c, z, x\}$, any 3-element subset of $\{a, b, c, x, y, z\}$ is $M$-independent.

Then there is a *-definable (over $M$ ) homogeneous space $(G, S)$ and generic (over $M)$ elements $a^{\prime}, b^{\prime}, c^{\prime}$ of $G$ and $x^{\prime}, y^{\prime}, z^{\prime}$ of $S$ such that $a^{\prime} \cdot x^{\prime}=y^{\prime}, b^{\prime} \cdot y^{\prime}=z^{\prime}$, and $c^{\prime} \cdot x^{\prime}=z^{\prime}\left(\right.$ so $\left.a^{\prime} \cdot b^{\prime}=c^{\prime}\right)$ such that each nonprimed element is interalgebraic over $M$ with the corresponding primed element.

Proof. The proof proceeds exactly as in the proof of Theorem 4.5 in [8]. The only possibly problematic step is the first one:

Lemma 2.2. There are *-tuples $a_{1}, b_{1}, c_{1}, x_{1}, y_{1}, z_{1}$ such that $a_{1}$ is interalgebraic over $M$ with a, etc., and moreover $y_{1} \in \operatorname{dcl}\left(M, b_{1}, z_{1}\right)$ and $z_{1} \in \operatorname{dcl}\left(M, b_{1}, y_{1}\right)$.

Proof. $b$ is first replaced by $b_{1}$ and $y$ by $y_{1}$ in such a way that $b$ is interalgebraic with $b_{1}$ over $M, y$ is interalgebraic with $y_{1}$ over $M$, and moreover whenever $\operatorname{tp}\left(z^{\prime} / M, b_{1}, y_{1}\right)=\operatorname{tp}\left(z / M, b_{1}, y_{1}\right)$ then $z$ and $z_{1}$ are interalgebraic over $M$. In the finitary case, $z$ is then replaced by the finite set $z_{1}$ of its $M, b_{1}, y_{1}$-conjugates, another imaginary element, interalgebraic with $a$ over $M$ and in $d c l\left(M, b_{1}, y_{1}\right)$. In the *-situation, the (in general infinite) set $X$ of $\left(M, b_{1}, y_{1}\right)$-conjugates of $z$ is not on the face of it another $*$-tuple. However, as pointed out in 7], $X$ can be identified with a $*$-tuple: let $z=\left(z_{i}\right)_{i \in I}$ say. For each finite $J \subset I$, let $z_{J}^{\prime}$ be the (finite) set of $M, b_{1}, y_{1}$-conjugates of the $J$-tuple $\left(z_{j}\right)_{j \in J}$, a single imaginary. Let $z_{1}$ be the *-tuple $\left(z_{J}^{\prime}\right)_{J}$. Then $z_{1}$ is interdefinable with $X$ (an automorphism fixes the $*$-tuple $z_{1}$ iff it fixes setwise the set $X$ of $*$-tuples). In particular $z_{1}$ is as required. The rest of the proof of the lemma proceeds in this way.

Proposition 2.3. Let $\bar{M}$ be a saturated model of a simple theory T. Suppose that $G, H$ are groups type-definable over a small model $M$ and that there are elements $a, b, c$ of $G$ and $a^{\prime}, b^{\prime}, c^{\prime}$ of $H$ such that:

(i) $a, b$ are generic independent over $M$,

(ii) $a \cdot b=c$ and $a^{\prime} \cdot b^{\prime}=c^{\prime}$,

(iii) a is interalgebraic with $a^{\prime}$ over $M$, and similarly for $b, b^{\prime}$ and $c, c^{\prime}$.

Then there is a type-definable over $M$ subgroup $G_{1}$ of bounded index in $G$ and a type-definable over $M$ subgroup $H_{1}$ of $H$, and a type-definable over $M$ isomorphism $f$ between $G_{1} / K_{1}$ and $H_{1} / L_{1}$ where $K_{1}$ is a finite normal subgroup of $G_{1}$, and $L_{1}$ is a finite normal subgroup of $H_{1}$.

Proof. Let $p=t p\left(a, a^{\prime} / M\right), q=t p\left(b, b^{\prime} / M\right)$ and $r=t p\left(c, c^{\prime} / M\right)$. Then $\left(a, a^{\prime}\right) \in$ $S(q, r)$. By Lemma $1.2 S(q, r)$ generates a type-definable over $M$ right coset $X$, say of $\operatorname{Stab}(r)$. The projection of $S t a b(r)$ on $G$ is $G_{M}^{0}$. By Lemma $1.2\left(a, a^{\prime}\right)$ is a generic point of $X$ over $M$. By definition, there is $\left(e, e^{\prime}\right) \in G \times H$, independent 
with $\left(a, a^{\prime}\right)$ over $M$ such that $\left(d, d^{\prime}\right)=\left(a, a^{\prime}\right)\left(e, e^{\prime}\right)^{-1}$ is a generic point of $\operatorname{Stab}(r)$ over $\left.M \cup\left\{e, e^{\prime}\right)\right\}$. As $a$ and $a^{\prime}$ are interalgebraic over $M, d$ and $d^{\prime}$ are interalgebraic over $M \cup\left\{e, e^{\prime}\right\}$. But $\left\{d, d^{\prime}\right\}$ is independent from $\left\{e, e^{\prime}\right\}$ over $M$, so $d$ and $d^{\prime}$ are interalgebraic over $M$. Thus both $\operatorname{Ker}(\operatorname{Stab}(q))=\{g \in G:(g, 1) \in \operatorname{Stab}(q)\}$ and $\operatorname{Coker}(\operatorname{Stab}(q))=\{h \in H:(1, h) \in \operatorname{Stab}(q)\}$ are finite normal subgroups of $G^{0}$ and $\pi_{2}(\operatorname{Stab}(q))$. This yields the proposition.

Remark 2.4. Suppose in the above that $T$ is supersimple and that $G, H$ are definable. Then by 11] $G_{1}$ can be chosen to be a definable subgroup of $G$ of finite index and $f$ can be chosen to be definable.

\section{Groups in DIFFERENCE FIELDS}

We will prove:

Theorem 3.1. Let $\bar{M}=(K,+, ., \sigma)$ be a model of $A C F A$. Let $G$ be a group definable in $\bar{M}$. Then there is a group $H$ definable in $(K,+,$.$) (i.e. an algebraic group),$ a definable subgroup $G_{1}$ of $G$ of finite index, a definable finite normal subgroup $N_{1}$ of $G_{1}$ and a definable embedding of $G_{1} / N_{1}$ into $H$.

Proof. We assume that $\bar{M}$ is saturated and work over a model $M$ over which $G$ is defined. Let $a, b, y$ be $M$-generic $M$-independent elements of $G$. Let $x=a . y$, $z=b^{-1} . y$ and $c=a . b$. Then $c . z=x$. Let $\bar{a}$ be the $*$-tuple $\left(\sigma^{i}(a): i \in \mathbf{Z}\right)$ and similarly for $\bar{b}, \bar{c}, \bar{x}, \bar{y}$ and $\bar{z}$. Then working in the field reduct $(K,+,$.$) ,$ $(\bar{a}, \bar{b}, \bar{c}, \bar{x}, \bar{y}, \bar{z})$ satisfy conditions (i) to (v) of Proposition 2.1. By that proposition there is in $(K,+,$.$) a *$-definable group over $M$, say $H$ with generic independent (over $M$ ) elements $a^{*}, b^{*}$, with $c^{*}=a^{*} \cdot b^{*}$ and such that, in $(K,+,),. a^{*}$ is interalgebraic with $\bar{a}$ over $M$, etc. By [4], $H$ is an inverse limit of groups $H_{i}$ $(i \in \omega)$, each of which is definable (in $(K,+,$.$) ), over M$. Let $\pi_{i}$ be the canonical surjective homomorphism from $H$ to $H_{i}$. Let $a_{i}=\pi_{i}\left(a^{*}\right)$ and similarly for $b_{i}, c_{i}$. So in $(K,+,),. a^{*}$ is interdefinable with $\left(a_{i}: i \in \omega\right)$ over $M$, and likewise for $b^{*}$ and $c^{*}$. In particular, in the structure $\bar{M}, a$ is interalgebraic with $\left(a_{i}: i \in \omega\right)$, and similarly for $b, c$. As $a, b, c$ are each finite tuples (and for $j<i, a_{j} \in d c l\left(M, a_{i}\right)$ ), there is some $i \in \omega$ such that $a$ is interalgebraic with $a_{i}, b$ with $b_{i}$ and $c$ with $c_{i}$ (all over $M$ ). Now apply Proposition 2.3 and Remark 2.4, and use the fact that $A C F$ has elimination of imaginaries.

Remark 3.2. The proof of Theorem 3.1 can be modified to show that the group configuration theorem (Proposition 2.1) holds for the theory ACFA (namely for any completion of ACFA). We prove a special case (Lemma 3.3) below. It is still unkown whether the group configuration theorem holds in arbitrary simple theories.

Lemma 3.3. Work in a saturated model $\bar{M}$ of $A C F A$, and let $M$ be a reasonably saturated submodel. Let $a, b, c, x, y, z$ be finite tuples from $\bar{M}$ such that (i), (iv), (v) from 2.1 hold, as well as

(ii) $^{\prime} \operatorname{acl}(M, a, x)=\operatorname{acl}(M, a, y)=\operatorname{acl}(M, x, y)$, and

(iii)' $\operatorname{acl}(M, b, z)=\operatorname{acl}(M, b, y)=\operatorname{acl}(M, z, y)$.

Then there is an $M$-definable group $G$ and $M$-generic $M$-independent elements $a^{\prime}, b^{\prime}$ of $G$, such that $a$ is interalgebraic with $a^{\prime}$ over $M, b$ is interalgebraic with $b^{\prime}$ over $M$ and $c$ is interalgebraic with $a^{\prime} . b^{\prime}$ over $M$. 
Proof. The proof of Theorem 3.1 yields an $M$-definable group $H$, and elements $a^{\prime}, b^{\prime}, c^{\prime}$ of $H$ such that $a^{\prime} \cdot b^{\prime}=c^{\prime}, a$ is interalgebraic with $a^{\prime}$ over $M, b$ is interalgebraic with $b^{\prime}$ over $M$ and $c$ is interalgebraic with $c^{\prime}$ over $M$. All we have to do is show that we can rechoose $a^{\prime}, b^{\prime}$ so as to be generic independent elements of some $M$-definable subgroup $G$ of $H$. Let $p=t p\left(a^{\prime} / M\right), q=t p\left(b^{\prime} / M\right)$ and $r=t p\left(c^{\prime} / M\right)$. Then $a^{\prime} \in S t(q, r)$. By Lemma 1.2 (ii), $p$ is a generic type of a left coset $X$ of $\operatorname{Stab}(q)$. Choose $a_{1}$ realizing $p$, independent from $\left\{a^{\prime}, b^{\prime}, c^{\prime}\right\}$ over $M$ such that $t p\left(a_{1}, c / M\right)=t p(a, c / M)$. Let $d=a_{1}^{-1} . a$, and $b_{1}=a_{1}^{-1} \cdot c^{\prime}$. Then $d . b^{\prime}=b_{1}$, $b_{1}$ realises $q$ and $\left\{d, b^{\prime}, b_{1}\right\}$ is pairwise independent over $M$. By Lemma 1.2 (iii), $q$ is a generic type of a right coset $Y$ of $\operatorname{Stab}(q)$. By [11], $\operatorname{Stab}(q)$ is the intersection of $M$-definable subgroups $G_{i}$. Choose $G=G_{i}$ with $S U\left(G_{i}\right)=S U(G)$. Let $X^{\prime}$ be the unique left translate of $G$ containing $X$, and $Y^{\prime}$ the unique right translate of $G$ containing $Y$. Note that $X^{\prime}, Y^{\prime}$ are $M$-definable sets, $p$ is a generic type of $X^{\prime}$ and $q$ a generic type of $Y^{\prime}$. Let $a^{\prime \prime} \in M \cap X^{\prime}$ and $b^{\prime \prime} \in M \cap Y^{\prime}$. Then $a_{1}=\left(a^{\prime \prime}\right)^{-1} \cdot a^{\prime}$ and $b_{1}=b^{\prime} \cdot\left(b^{\prime \prime}\right)^{-1}$ are $M$-generic elements of $G$, interdefinable with $a^{\prime}, b^{\prime}$ respectively over $M$. Moreover $c_{1}=a_{1} . b_{1}$ is interdefinable with $c^{\prime}$ over $M$. So $G$ and $a_{1}, b_{1}$ work.

Remark 3.4. The Zariski-dense subgroups of simple algebraic groups, definable in models of $A C F A$, are classified in [3]. So with Theorem 3.1 this yields a classification of simple groups definable in models of $A C F A$.

\section{The DifFEREntial CASE REVISITED}

The following was proved in 9].

Theorem 4.1. Let $M=(K,+, ., D)$ be a differentially closed field of characteristic 0 . Let $G$ be a connected group definable in $M$. Then there is a connected group $H$ definable in $(K,+,$.$) and a definable (in M$ ) embedding of $G$ in $H$.

The proof (on which the proof of Theorem 3.1 is modelled) involved embedding $G$ in a group $G^{\infty}$ which is *-definable in $A C F$, then appealing to the fact that $G^{\infty}$ is an inverse limit of groups definable in $A C F$ (algebraic groups) as well as to the $D C C$ for differential algebraic groups, so as to embed $G$ in an algebraic group. The use of the $D C C$ was unnecessary. In fact the construction of $G^{\infty}$ also turns out to be unnecessary, and we will sketch here a rather more direct proof of Theorem 4.1. (However the new proof involves the same machinery used to prove that a $*$-definable group is an inverse limit of type-definable groups, but applied only once.)

Sketch of the proof of Theorem 4.1. We may assume $M$ is saturated. Let $k$ be a small model over which $G$ is defined. Let $p(x) \in S(k)$ be the generic type of $G$. Let $a$ realise $p(x)$ and let $p^{*}\left(x^{*}\right)$ be the complete type of $a^{*}=\left(a, D(a), D^{2}(a), \ldots\right)$ over $k$ in the algebraically closed field $(K,+,$.$) . As definable functions in D C F_{0}$ are differential rational, for independent realizations $a, b$ of $p(x),(a . b)^{*}$ is contained in $k\left(a^{*}, b^{*}\right)$, and so is of the form $f\left(a^{*}, b^{*}\right)$ for some $*$-definable function over $k$ in $A C F$. Note that $(a . b)^{*}$ realises $p^{*}$ and that $a^{*}, b^{*},(a, b)^{*}$ are pairwise independent over $k$ in $A C F$. Now suppose $c, d$ are independent over $k$ realizations of $p^{*}$ (in $A C F)$. So in $A C F \operatorname{tp}(c, d / k)=t p\left(a^{*}, b^{*} / k\right)$ whereby $f(c, d)$ is defined and also realises $p^{*}$, independently from each of $c, d$. We write $f(c, d)$ as $c . d$, hopefully without ambiguity. Note that for independent $c, d, e$ realising $p^{*}$ (in $A C F$ ) we have 
$(c . d) . e=c .($ d.e $)$ realises $p^{*}$. We write $c$.d.e for this. We also write a realization $c$ of $p^{*}$ as $\left(c_{0}, c_{1}, \ldots\right)$ (corresponding to the enumeration $a^{*}=(a, D(a), \ldots)$ ).

We now work in $A C F$. Define the following equivalence relation $E$ on realizations of $p^{*}: E\left(c, c^{\prime}\right)$ if for some (any) realisations $d, e$ of $p^{*}$ such that $d$ and $e$ are generic, independent over $k \cup\left\{c, c^{\prime}\right\}$, $(\text { d.c.e })_{0}=\left(d . c^{\prime} . e\right)_{0}$. By definability of types in $A C F$, $E$ is $*$-definable over $k$.

Claim 1. For $c, d k$-independent realizations of $p^{*}$, each of $c / E, d / E, c . d / E$ depends only on the other two.

Proof. We show that if $E\left(c, c^{\prime}\right)$, where $c^{\prime}$ realizes $p^{*}$ and is independent from $d$ over $k$, then $c . d / E=c^{\prime} . d / E$. Choose $e, f$ realizing $p^{*}$ independent over everything; then $e$ and $d . f$ are realizations of $p^{*}$ independent of $c, c^{\prime}$ over $k$. Hence $(e . c .(d . f))_{0}=$ $\left(e . c^{\prime} .(d . f)\right)_{0}$. So $(e .(c . d) . f)_{0}=\left(e .\left(c^{\prime} . d\right) . f\right)_{0}$ and $c . d / E=c^{\prime} . d / E$. This proves that c.d/E depends on $c / E$ and $d / E$. The rest of the claim follows similarly.

Claim 2. Let $c, c^{\prime}$ realize $p^{*}$. Then $E\left(c, c^{\prime}\right)$ implies $c_{0}=c_{0}^{\prime}$.

Proof. Choose $d, e$ generic independent over $c, c^{\prime}$ realizations of $p^{*}$. Clearly $c=d . b . e$ for some realization $b$ of $p^{*}$ independent with $d, e$ over $k$, and likewise $c^{\prime}=d \cdot b^{\prime} . e$ for suitable $b^{\prime}$ realising $p^{*}$. As $E\left(c, c^{\prime}\right)$, by Claim 1 we get $E\left(b . e, b^{\prime} . e\right)$ and again by Claim $1, b / E=b^{\prime} / E$. As $d, e$ are generic independent realizations of $p^{*}$ over $b, b^{\prime}$ it follows that $c_{0}=c_{0}^{\prime}$ as required.

Claim 3. Let $c$ realise $p^{*}$. Then for some $n<\omega, c / E$ depends only on $\left(c_{0}, \ldots, c_{n}\right)$.

Proof. Let $d, e$ be independent realizations of $p^{*}$ over $k, c$. Then the finite tuple $(\text { d.c.e })_{0}$ is in $k(d, c, e)$, so in $k\left(d_{0}, \ldots, d_{n}, c_{0}, \ldots, c_{n}, e_{0}, \ldots, e_{n}\right)$ for some $n$. So clearly $c / E \in k\left(c_{0}, \ldots, c_{n}\right)$.

By Claim 3 let $c^{\prime}$ be a finite tuple such that $c^{\prime}$ is interdefinable with $c / E$ over $k$ (in $A C F)$. Let $p^{\prime}(x)=t p\left(c^{\prime} / k\right)$ in $A C F$. By Claim 1, the operation . on realisations of $p^{*}$ induces an operation $f(-,-)$ on independent realisations of $p^{\prime} . f$ is definable over $k$ in $A C F$ and generically associative. By Weil's theorem (or the more general version due to Hrushovski [4]), $p^{\prime}$ is the generic type of a $k$-definable connected group (in $A C F$ ), $H$ say, and $f$ agrees with multiplication in $H$. The map taking a realization $a$ of $p$ to $\left(a^{*}\right)^{\prime}$ (definable in $D C F$ over $k$ ) is 1-1 by Claim 2, and so extends to a definable (in $D C F$ ) embedding of $G$ into $H$.

\section{Difference Algebraic groups on AFFine SPACES}

In this final section we briefly point out that results from [6] and [1] pass over to the difference context.

Let $(K,+, ., \sigma)$ be a model of $A C F A$ and let $K\left\{X_{1}, \ldots, X_{n}\right\}$ be the difference ring of difference polynomials over $K$ in difference indeterminates $X_{1}, \ldots, X_{n}$.

Definition 5.1. (i) By an affine difference variety we mean a subset $X$ of $K^{n}$ which is the zero set of a finite number of difference polynomials.

(ii) By a morphism between affine difference varieties $X \subseteq K^{n}$ and $Y \subseteq K^{m}$ we mean a map $\left(f_{1}, \ldots, f_{m}\right)$ from $X$ to $Y$ where each $f_{i}$ is the restriction to $X$ of a differential polynomial in $K\left\{X_{1}, \ldots, X_{n}\right\}$.

(iii) By an affine difference algebraic group we mean a group $G$ such that the underlying set of $G$ is an affine difference variety and both multiplication and inversion are morphisms. 
Remark 5.2. It would be more appropriate to define a morphism to be something which is locally given by a difference rational function. However we will be interested here in the case where the underlying set of $G$ is $K^{n}$ and in this case the notions coincide.

Theorem 5.3. Suppose $G$ is a difference algebraic group whose underlying set is $K^{n}$. Then $G$ is embeddable, by a morphism of difference algebraic groups, in a linear unipotent algebraic group $H$ over $K$.

Proof sketch. For $a \in G$ (namely in $K^{n}$ ) let $a^{*}=\left(a, \sigma(a), \sigma^{2}(a), \ldots\right)$. Let $X_{i}, Y_{i}$ denote $n$-tuples of indeterminates. Then as in [6] there are polynomial functions $f_{i}\left(X_{0}, X_{1}, \ldots, Y_{0}, Y_{1}, \ldots\right)$ and $g_{i}\left(X_{0}, X_{1}, \ldots\right)$ over $K(i<\omega)$ such that

(i) there is a group $G^{\infty}$ on infinite dimensional affine space $A^{\infty}$ over $K$, whose multiplication is defined by $\left(f_{i}\right)_{i}$ and inversion by $\left(g_{i}\right)_{i}$.

(ii) for $a, b \in K^{n}$,

$$
(a . b, \sigma(a . b), \ldots)=\left(f_{0}\left(a^{*}, b^{*}\right), f_{1}\left(a^{*}, b^{*}\right), \ldots\right),
$$

and similarly for inversion.

By Theorem 2 of [1, $G^{\infty}$ is isomorphic, as a group scheme over $K$ to an inverse limit $\left(G_{i}\right)_{i<\omega}$ of linear unipotent connected algebraic groups over $K$. Let $\mu_{i}$ be the canonical surjective homomorphism from $G^{\infty}$ to $G_{i}$.

The map $h$ taking $a$ to $a^{*}$ is an embedding of $G$ into $G^{\infty}$, quantifier-free definable in $(K,+, ., \sigma)$. Composing with the $\mu_{i}$ gives quantifier-free definable homomorphisms $h_{i}: G \rightarrow G_{i}$. The intersection of all the $\operatorname{Ker}\left(h_{i}\right)$ is the identity of $G$. On the other hand, each $\operatorname{Ker}\left(h_{i}\right)$ is quantifier-free definable in $(K,+, \sigma)$, so by the $D C C$ on quantifier-free definable subgroups, some $h_{i}$ is an embedding, as required.

Remark 5.4. (i) Clearly $(K,+, ., \sigma)$ can be an arbitrary difference field (not necessarily a model of $A C F A$ ) above.

(ii) What can be said about groups definable in a model $(K,+, ., \sigma)$ whose underlying set is some $K^{n}$ ? (Namely the group operation is definable, but not necessarily by a difference polynomial function.)

\section{REFERENCES}

[1] M. Chalupnik and P. Kowalski, Lazard's theorem for differential algebraic groups and proalgebraic groups on affine spaces, preprint 1999.

[2] Z. Chatzidakis and E. Hrushovski, Model theory of difference fields, Transactions of AMS, 351 (1999), 2997-3071. MR 2000f:03109

[3] Z. Chatzidakis, E. Hrushovski and Y. Peterzil, The model theory of difference fields II: periodic ideals and the trichotomy theorem in all characteristics, submitted to Proceedings of London Math. Soc.

[4] E. Hrushovski, Unidimensional theories are superstable, Annals of Pure and Applied Logic, 50 (1990), 117-138. MR 92g:03052

[5] E. Hrushovski and A. Pillay, Groups definable in local fields and pseudo-finite fields Israel Journal of Math., 85 (1994), 203-262. MR 95f:12015

[6] P. Kowalski and A. Pillay, Pro-algebraic and differential algebraic group structures on affine spaces, American Journal of Mathematics, 1222 (2000), 213-221. CMP 2000:07

[7] D. Lascar and A. Pillay, Hyperimaginaries and automorphism groups, to appear in Journal of Symbolic Logic.

[8] A. Pillay, Geometric Stability Theory, Oxford University Press, 1996. MR 98a:03049

[9] A. Pillay, Some foundational questions on differential algebraic groups, Pacific Journal of Math., 179 (1997), 179-200. MR 98g:12008 
[10] A. Pillay, Definability and definable groups in simple theories, Journal of Symbolic Logic, 63 (1998), 788-796. MR 2000a:03055

[11] F. O. Wagner, Groups in simple theories, preprint 1999.

Department of Mathematics, University of Wroclaw, pl Grunwaldzki 2/4, 50-384 Wroclaw, Poland

E-mail address: pkowa@math.uni.wroc.pl

Department of Mathematics, University of Illinois at Urbana-Champaign, Urbana, ILLINOIS 61801

E-mail address: pillay@math.uiuc.edu 